\title{
Evaluation of a novel approach for the measurement of RNA quality
}

\author{
Timothy M Wilkes*, Alison S Devonshire, Stephen LR Ellison, Carole A Foy
}

\begin{abstract}
Background: Microarray data interpretation can be affected by sample RNA integrity. The ScreenTape Degradation Value (SDV) is a novel RNA integrity metric specific to the ScreenTape ${ }^{\oplus}$ platform (Lab901). To characterise the performance of the ScreenTape ${ }^{\circledast}$ platform for RNA analysis and determine the robustness of the SDV metric, a panel of intentionally degraded RNA samples was prepared. These samples were used to evaluate the ScreenTape ${ }^{\circledast}$ platform against an alternative approach for measuring RNA integrity (Agilent Bioanalyzer RIN value). The samples were also subjected to microarray analysis and the resulting data correlated to the RNA integrity metrics.

Findings: Measurement of SDV for a panel of intentionally degraded RNA samples ranged from 0 for intact RNA to 37 for degraded RNA, with corresponding RIN values ranging from 10 to 4 for the same set of samples. SDV and RIN scales both demonstrated comparable discrimination between differently treated samples (RIN 10 to 7, SDV 0 to 15), with the SDV exhibiting better discrimination at higher degradation levels. Increasing SDV values correlated with a decrease in microarray sample labelling efficiency and an increase in numbers of differentially expressed genes.

Conclusions: The ScreenTape ${ }^{\oplus}$ platform is comparable to the Bioanalyzer platform in terms of reproducibility and discrimination between different levels of RNA degradation. The robust nature of the SDV metric qualifies it as an alternative metric for RNA sample quality control, and a useful predictor of downstream microarray performance.
\end{abstract}

\section{Background}

The use of microarray technology has revolutionised the fields of molecular biology and genetics. However, concerns have been raised over the numerous potential sources of variation that can affect assay consistency and data quality [1,2]. Previous studies have highlighted RNA integrity as one source that has a major effect on microarray data quality [3-5].

To date, no single RNA integrity metric has been adopted universally by the research community. RNA quality is commonly determined by several different techniques, including the ribosomal peak ratio (eukaryotic 28s/18s rRNA peak intensity ratio) [6], RIN [7], 5'/3' transcript signal intensity ratio determined by qRT-PCR or microarray analysis [8] and other quality indices [9].

RNA purity is assessed routinely by measuring the OD260 $\mathrm{nm} / \mathrm{OD} 280 \mathrm{~nm}$ ratio $[6,10,11]$ of a sample. However, this metric yields no information about RNA integrity. Molecular biologists have therefore relied on the technique of gel electrophoresis, which provides a

\footnotetext{
* Correspondence: TIMOTHY.WILKES@LGC.CO.UK

LGC, Queens Road, Teddington, Middlesex, TW11 OLY, UK
}

reproducible separation of ribosomal RNA (rRNA) molecules to derive overall sample RNA integrity. Currently, such conventional methods are being replaced by microfluidic-based platforms, such as that developed by Agilent Technologies (2100 Bioanalyzer).

More recently, Lab901 have developed a novel electrophoretic ScreenTape ${ }^{\circledR}$ platform that employs precast multilane gels and microfluidics enabling semi-automated operation, simplifying sample handling and reducing assay times. In this study we have compared the performance characteristics of the ScreenTape ${ }^{\circledR}$ R6K platform (Lab901) and corresponding RNA quality metric, SDV [9] with the 2100 Bioanalyzer and associated quality metric, RIN [7].

We report here on the broad correlation observed for these quality metric values and associated microarray data.

\section{Methods \\ RNA preparation}

HepG2 cells (passage $=84$ ) were grown to confluence in T175 vented culture flasks, using Eagles Minimum Essential Medium (EMEM, ATCC) plus 10\% Foetal Calf 
Serum (FCS, Invitrogen) in a humid $37^{\circ} \mathrm{C}$ incubator supplemented with $5 \% \mathrm{CO}_{2}$. Cells were then exposed for $24 \mathrm{~h}$ to EMEM exposure media supplemented with $0.5 \%$ (v/v) DMSO vehicle (Sigma Aldrich) and $4 \mathrm{mM} \mathrm{ACAP}$ (Paracetamol, Sigma Aldrich). Following treatment, the cells were washed with an excess of $1 \times$ Phosphate Buffered Saline (Gibco) before being lysed in situ by the application of ice cold TRIzol ${ }^{\circledR}$ LS Reagent (Invitrogen). Total RNA was then isolated according to manufacturer's instructions (Invitrogen). RNA quantity was determined using a NanoDrop 1000 spectrophotometer.

\section{Instrumentation}

For comparative analysis of RNA integrity, the Tape Station ${ }^{\circledR}$ (Lab901) was used in conjunction with ScreenTape ${ }^{\circledR}$ R6K, and the 2100 Bioanalyzer (Agilent Technologies) with the RNA 6000 series II Nano LabChip analysis kit. Total RNA samples were prepared for analysis according to manufacturer's recommendations. Results were compared between the platforms for six levels of RNA sample integrity and at a single RNA concentration of $25 \mathrm{ng} / \mu \mathrm{l}$.

\section{RNA degradation}

RNA from the treated HepG2 cells was diluted to a concentration of $1 \mu \mathrm{g} / \mu \mathrm{l}$ with nuclease-free water (Ambion) and aliquoted into volumes of $50 \mu \mathrm{l}$ in $0.2 \mathrm{ml}$ thin wall PCR tubes. The tubes were placed in the block of an MJ Research PTC-200 DNA Engine Thermal Cycler PCR machine which was then heated to $90^{\circ} \mathrm{C}$. Tubes were removed in batches of three at 3-minute intervals. The RNA was then diluted to $25 \mathrm{ng} / \mu \mathrm{l}$ with nuclease-free water, and RNA integrity for each sample determined on both platforms.

\section{Microarray experimental design}

A single-colour labelling approach was adopted for the microarray hybridisation scheme. Duplicate biological samples of those employed for the Lab901/Bioanalyser platform evaluation were used, with two technical replicates (arrays) for each sample. Samples were hybridized to Agilent Homo sapiens $4 \times 44 \mathrm{~K}$ whole genome gene expression arrays according to manufacturer's instructions. The hybridised arrays were scanned using an Agilent G2505B Scanner and expression data extracted using Feature Extraction software, version 10.5 (Agilent Technologies). Data was exported to the Genespring GX (Agilent) software package, normalised to the 75th percentile of the data set and base-lined to the median signal intensity of all chips. The data were quality controlled by filtering on flags (features present and marginal), before the number of differentially expressed genes (DEG) were determined using the combination of an analysis of variance (ANOVA) and relative fold change $(\mathrm{FC}=>1.5)$.

\section{Statistical treatment}

All statistical analysis used $R$ version 2.9.2 [12].

\section{Results and discussion \\ RNA Integrity Measurements}

RNA integrity was assessed for RNA samples with increasing levels of degradation using the Lab901 ScreenTape ${ }^{\circledR}$ system, which generates SDV values, and the Agilent Bioanalyzer, which generates RIN values. Replicate samples were run on three separate chips or tapes, depending on the platform, and across triplicate lanes per chip or tape. An overlay of chromatograms generated by the ScreenTape ${ }^{\circledR}$ and Bioanalyzer systems for intact and degraded RNA is presented in Figure 1.

\section{Comparison with RNA Integrity Number (RIN)}

RIN is an incremental scale which spans from 0 to 10 , with increasing RNA integrity correlating with increasing RIN value. In contrast, SDV is an unconstrained metric which employs a scale of measurement spanning from 0 to infinity with higher values corresponding to increased degradation. A direct comparison of SDV to RIN was carried out to evaluate the performance of the SDV metric. Preliminary inspection of the data for both platforms (Figure 2) illustrates several important features:

(i) Both RIN and SDV show good discrimination between samples in general.

(ii) SDV appears to show generally better withingroup precision.

(iii) There is evidence to suggest that RIN and SDV do not share a linear relationship.

(iv) The within-group dispersion does not appear to be constant across treatment groups for either of the metrics.

\section{Performance comparison}

The assessment of relative platform performance was determined by the use of three different statistical indicators, namely rank order correlation, intraclass correlation and classification performance

\section{i) Rank order correlation}

Spearman $(\rho)$ and Kendall $(\tau)$ rank correlations between response and RNA integrity level are listed in Table 1. Both correlation measures indicate very good performance, with the SDV performance appearing marginally better. However, based upon bootstrapping studies (5000 resamples with 95\% BCa confidence interval [13] calculated for the difference in magnitude), the difference in rank correlation is not significant at the $95 \%$ level of confidence. 


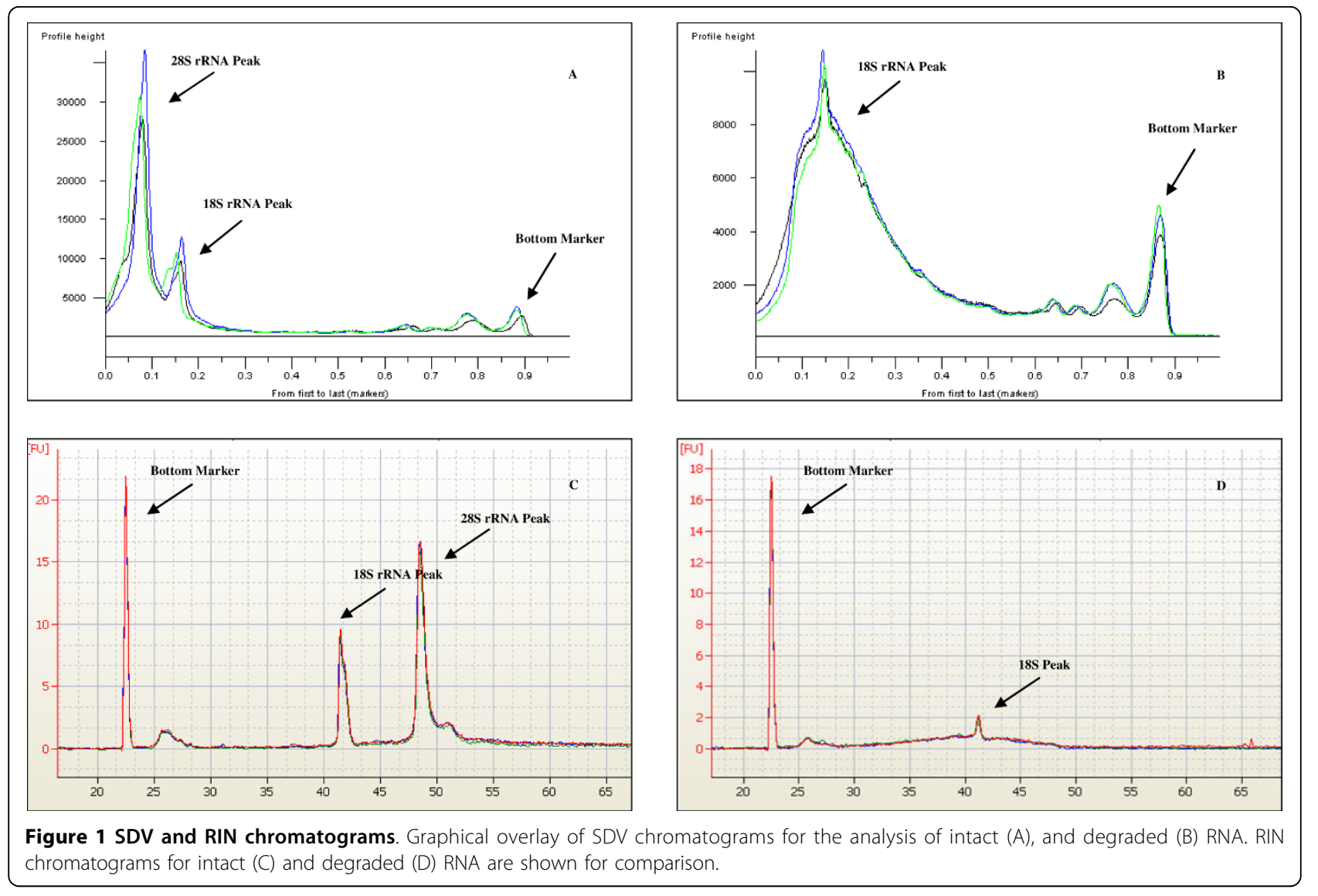

\section{ii) Intraclass correlation}

Table 2 lists the calculated intraclass correlation coefficients (ICC) for each metric with respective calculated confidence intervals.

The ICC for both metrics are high, which is indicative of good performance. The observed ICC for SDV is marginally higher but the confidence intervals show that the difference is not significant at the $95 \%$ level of confidence.

\section{iii) Classification performance}

Four supervised classification methods (rank ordering, Gaussian, K-nearest neighbour (KNN) and linear discrimination analysis (LDA)) were applied to the data sets and the resulting groupings compared with the expected groupings. The significance for differences in the proportion of misclassifications between the RIN and SDV metrics were then tested for each of the classification methods employed. Table 3 summarises these results in terms of the number of misclassifications observed and associated $p$-values.

It can be seen that the relative performance of the two RNA degradation metrics is consistent across all four classification methods. However, more misclassifications were observed when using the RIN metric. In addition, the difference in misclassification was found to be significant at the $95 \%$ level for two of the four classification methods. This would indicate that a significant difference in performance exists between the SDV and RIN metrics.

\section{Microarray analyses}

\section{RNA integrity and labelling efficiency}

Gene expression studies are potentially sensitive to the effect of RNA integrity. To determine the broad impact of RNA integrity on microarray data, and to determine the value of the SDV metric in highlighting potential problems, microarray labelling data was generated for the degraded RNA samples used in this study. The procedure recommended by Agilent for the amplification and labelling of mRNA samples employs a 3' mediated Eberwine amplification protocol [14] that introduces a directional bias into the pool of synthesised cRNA. Progressive degradation of the RNA template will reduce the population fragment size and is expected to lead to a reduced labelling efficiency. Plotting of the labelling data demonstrates that reduced RNA integrity leads to reduced sample labelling (Cyanine 3 ( $\mathrm{pmol} / \mu \mathrm{g}$ RNA)) (Figure 3).

In terms of microarray assay performance, reduced sample labelling efficiency may have an impact on the 


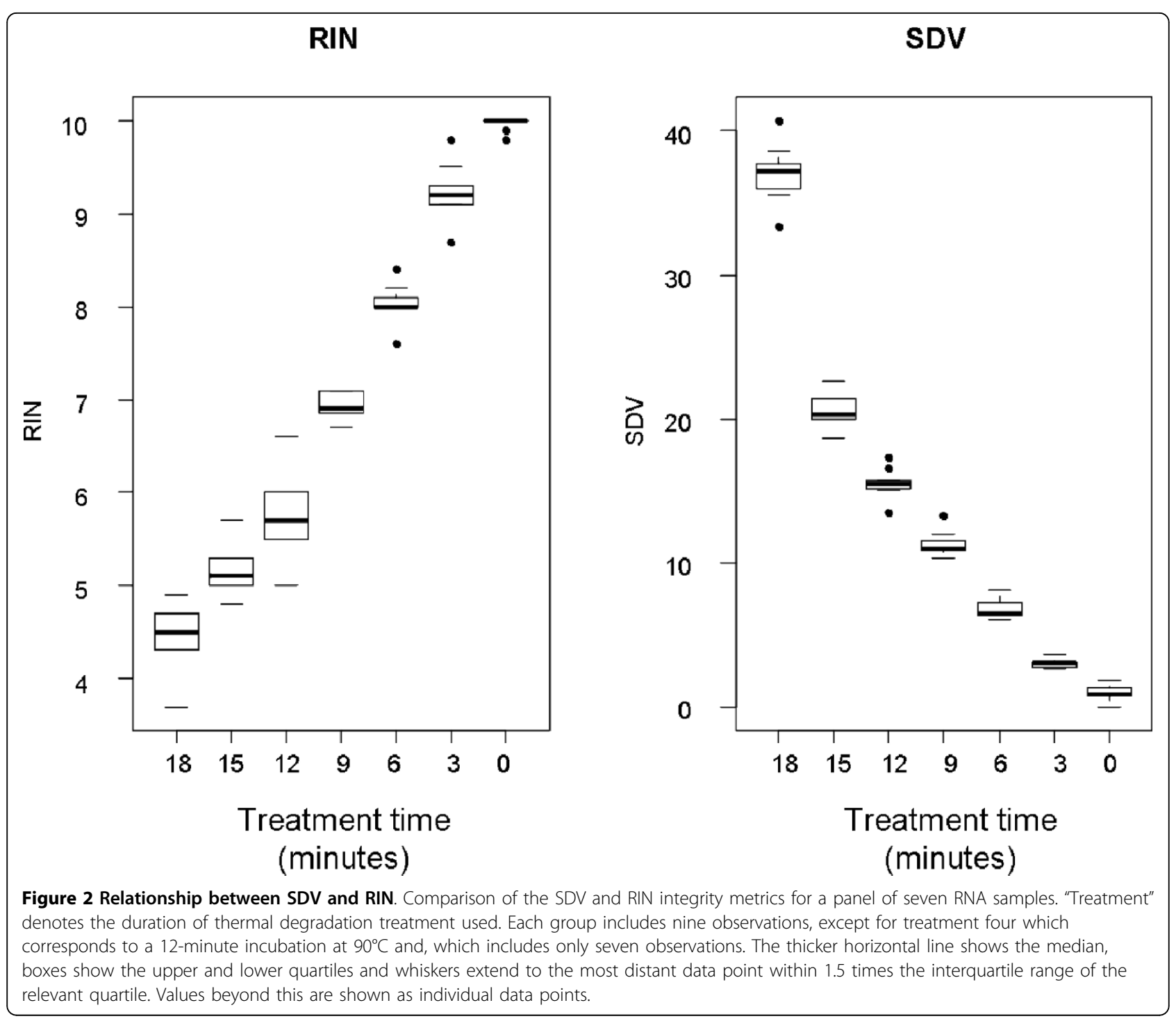

Table 1 Rank correlations (observed vs. treatment level)

\begin{tabular}{lll}
\hline & Spearman $\boldsymbol{\rho}$ & Kendall $\boldsymbol{\tau}$ \\
\hline RIN & 0.984 & 0.927 \\
SDV & -0.990 & -0.934 \\
\hline
\end{tabular}

Spearman $(\rho)$ and Kendall $(\tau)$ rank correlations between observed RNA integrity level and degradation treatment time.

Table 2 Intraclass correlation coefficients

\begin{tabular}{lll}
\hline & ICC $^{*}$ & 95\% Confidence interval \\
& ** \\
\hline RIN & 0.981 & $(0.949,0.997)$ \\
SDV & 0.992 & $(0.979,0.998)$ \\
\hline
\end{tabular}

* Intraclass correlation coefficient ICC(1) from reference [15]

** Calculated as in reference [15]

Calculated intraclass correlation coefficients with associated calculated confidence intervals. robustness of microarray measurement and data reliability. RNA integrity measurement, whether by SDV or RIN, may provide a valuable tool for early prediction of RNA labelling efficiency and ultimately of overall microarray performance.

\section{Microarray data}

Assay sensitivity (the rate of detection of true positives) and specificity (the rate of detection of true negatives) are important performance indicators for microarray experiments. To gain insight into the broad impact of RNA integrity, as determined by SDV, a preliminary gene expression experiment was performed using the samples from this study. For each data set generated using different levels of RNA integrity, the number of differentially expressed genes (DEGs) was determined by 
Table 3 Misclassification rates

\begin{tabular}{llll}
\hline Classification method & RIN & SDV & p-value \\
\hline Sample ranking & $8 / 61$ & $0 / 63$ & 0.009 \\
Gaussian classifier & $7 / 61$ & $1 / 63$ & 0.061 \\
KNN & $7 / 61$ & $0 / 63$ & 0.017 \\
LDA & $8 / 61$ & $2 / 63$ & 0.089 \\
\hline
\end{tabular}

Table shows the number of misclassified observations and the total number of observations (two observations were missing from the 9-minute treatment set). All the observed misclassifications were found to be assigned to adjacent treatment groups. Note that for $\mathrm{KNN}$, the misclassification rate varies from run to run due to random tie-breaking (values from 6 to 8 were observed for the 9-minute treatment); the value of 7 given here for KNN was the modal value for $100 \mathrm{KNN}$ reruns.

comparing the paracetamol-treated HepG2 cells to intact RNA extracted from HepG2 cells treated with vehicle control only (DMSO). To assign a measure of assay specificity to the data, a value for the degree of additional gene discovery ("Additional Discovery Rate", ADR) at each level of RNA integrity was derived by subtracting the median number of Differentially Expressed Genes (DEGs) for the technical replicates of the intact sample from the median number of DEGs determined for each of the degraded samples (Table 4).
Differential expression was defined as those genes which demonstrated a statistically significant difference of expression $(\mathrm{p} \leq 0.05)$ while employing a Benjamini and Hochberg [15] false discovery rate correction factor, and a fold change $\geq 1.5$ compared to the control. The additional gene discovery rate was determined by subtracting the median number of genes determined for three technical replicates of an intact RNA sample away from the median number of genes derived from three technical replicates at each level of degraded RNA sample.

It has been reported previously that gene expression profiling using Affymetrix GeneChip arrays is relatively tolerant to moderate RNA degradation as well as to 5' truncation occurring as a consequence of successive rounds of in vitro transcription [16]. However, with progressively decreasing RNA integrity, a substantial increase in the rate of detection of additional positives is reported here, particularly with RIN values $<7$ [3]. Comparing RIN or SDV with the ADR reveals a progressive increase in the ADR with decreasing RNA integrity. The exact nature of this increase is uncertain at this stage, but these findings indicate that a shift in

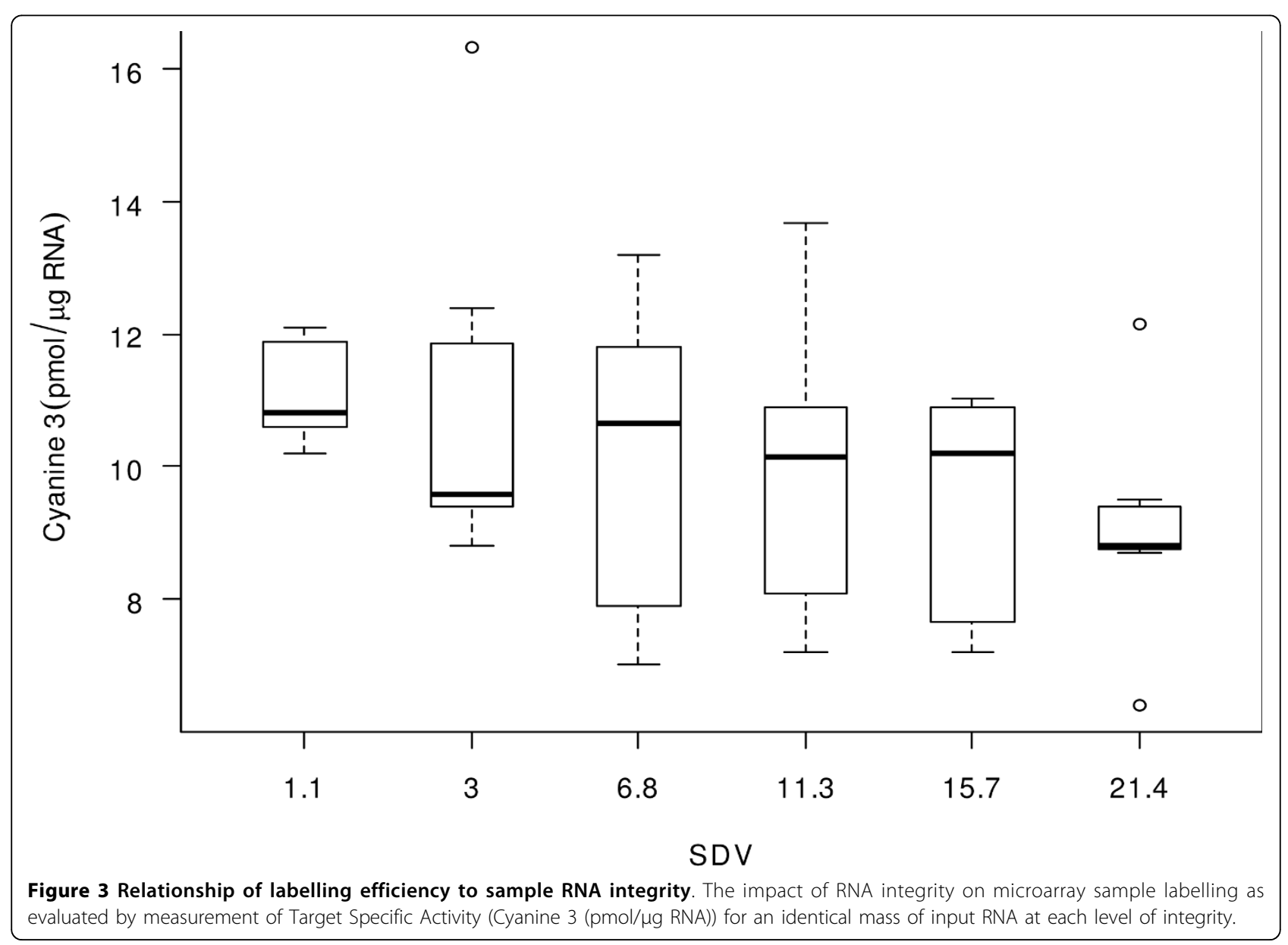


Table 4 The number of differentially expressed genes and associated additional gene discovery rate at each level of RNA integrity

\begin{tabular}{ccccc}
\hline Treatment Time $(\mathbf{m i n})$ & Measured RIN & Measured SDV & Median number of genes detected* & Additional Discovery Rate \\
\hline 0 & 10 & 1.3 & 5948 & 0 \\
\hline 3 & 9.2 & 2.9 & 7509 & 1561 \\
\hline 6 & 7.8 & 6.5 & 8896 & 2948 \\
\hline 9 & 7 & 11.2 & 10129 & 4181 \\
\hline 12 & 5.5 & 15.4 & 10621 & 4673
\end{tabular}

* Genes demonstrating a statistical difference from DMSO sample $(P \leq 0.05)$ and with a fold change $\geq 1.5$

assay specificity is occurring as a consequence of reduced RNA integrity, which can be accurately measured and predicted with SDV and RIN.

\section{Conclusions}

The measurement of gene expression is based on the assumption that an analysed RNA sample accurately represents the population of transcripts present in vivo [17]. Many transcripts demonstrate stability differences of several orders of magnitude in vivo [18], raising the possibility that partial sample degradation could cause variable bias in transcript quantification. The adoption of a suitable RNA quality metric with the capacity to accurately determine RNA integrity is therefore an essential prerequisite for robust data generation in any expression profiling experiment.

In this paper we have compared both the performance of the novel Lab901 ScreenTape ${ }^{\circledR}$ platform with that of the Agilent 2100 Bioanalyzer and also compared the SDV metric with the RIN metric for the determination of RNA integrity when applied to microarray data analysis.

Both metrics performed well when using the samples employed in this study, with the data highlighting the difficulty associated with unambiguously assigning samples to a definitive integrity level when they have ether a RIN value $\leq 6$ or an SDV value $\geq 15$.

In conclusion, the ScreenTape ${ }^{\circledR}$ system was demonstrated to be a reliable and robust means of determining RNA integrity with SDV estimates, correlating well with the RIN values generated by the Agilent Bioanalyzer platform and with a better classification performance in this study. In addition, the RIN and SDV metrics both performed well in terms of distinguishing different levels of RNA degradation treatment. For microarray data, Rank correlations with treatment and intraclass correlations are high. Classification methods show that the majority of observations were classified into appropriate treatment groups by both RIN and SDV, with the slightly better classification performance of the SDV metric being significant at the $\mathrm{P} \leq 0.05$ level for two out of the four classification methods used.
The ScreenTape platform and SDV therefore offer an alternative to currently available systems for RNA integrity analysis and provide a performance comparable to that of the Bioanalyzer 2100. The rapid assay time and medium through put capacity may favour its use in laboratories which process large numbers of samples. Future studies with this platform could enable the development of an SDV based RNA quality threshold to be established for use with downstream applications.

\section{Acknowledgements}

The work described in this paper was undertaken by LGC in its capacity as the designated National Measurement Institute. This work was funded by the UK National Measurement System under the Measurement for Innovators Programme and, in part, by a financial contribution made by Lab901, as required by the Programme.

\section{Authors' contributions}

TW participated in the design of the study, carried out the platform evaluations and drafted the manuscript, AD carried out the RNA degradation and SE performed the statistical analyses. CF conceived of the study and participated in its design and coordination and helped to draft the manuscript. All authors read and approved the final manuscript.

\section{Competing interests}

The authors declare that they have no competing interests.

Received: 22 December 2009 Accepted: 1 April 2010

Published: 1 April 2010

\section{References}

1. Hessner MJ, Meyer L, Tackes J, Muheisen S, Wang X: Immobilised probe and glass surface chemistry as variables in microarray fabrication. BMC Genomics 2004, 5:53.

2. Jarvinen AK, Hautaniemi S, Edgren $H$, Auvinen P, Sarrela J, Kallioiemi OP Monni O: Are data from different gene expression microarrays comparable? Genomics 2004, 83:1164-8

3. Thompson $\mathrm{KL}$, Scott Pine $\mathrm{P}$, Rosenzweig BA, Turpaz $Y$, Retief Characterization of the effect of sample quality on high density oligonucleotide microarray data using progressively degraded rat liver RNA. BMC Biotechnology 2007, 7:57

4. Copois V, bibeau F, Bascoul-Mollevi C, Salvetat N, Chabos P, Bareli C, Candeil L, Fraslon C, Conseiller E, Granci V, Maziere P, Kramar A, Ychou M, Pau B, Martineau P, Molina F, Del Rio M: Impact of RNA degradation on gene expression profiles: Assessment of different methods of reliability determines RNA quality. Journal of Biotechnology 2007, 127:549-559.

5. Grissom SF, Lobenhofer EK, Tucker CJ: A qualitative assessment of direct labelled cDNA products prior to microarray analysis. BMC Genomics 2005, 6:36.

6. Sambrook J, Fritsch EF, Maniatis T: Molecular Cloning: A Laboratory Manual. Cold Spring Harbour, 21989

7. Schroeder A, Mueller O, Stocker S, Salowsky R, Leiber M, Gassman M, Lightfoot S, Menzel W, Granzow M, Ragg R: The RIN: An RNA integrity 
number for assigning integrity values to RNA measurements. BMC

Molecular Biology 2006, 7:3.

8. Finkelstein DB: Trends in the Quality of Data from 5168 Oligonucleotide Microarrays from a Single Facility. Journal of Biomolecular Techniques 2005, 16:143-153.

9. Auer H, Lyianarachchi S, Newsom D, Kilsovic MI, Marcucci G, Kornacker K: Chipping away at the chip bias: RNA degradation in microarray analysis. Nat Genet 2003, 35(4):292-293.

10. Manchester KL: Value of A260/A280 ratios for measurement of purity of nucleic acids. Biotechniques 1995, 19:208-210.

11. Manchester KL: Use of UV methods for measurement of protein and nucleic acid concentrations. Biotechniques 1996, 20:968-970.

12. R Development Core Team: R: A language and environment for statistical computing. R Foundation for Statistical Computing, Vienna, Austria 2008 [http://www.R-project.org], ISBN 3-900051-07-0.

13. Davison AC, Hinkley DV: Bootstrap methods and their application. Cambridge University Press 1997, chapter 5.

14. Eberwine J, Yeh $H$, Miyashiro K, Cao Y, Nair S, Finnell R, Zettel M, Coleman P: Analysis of gene expression in single live neurons. PNAS 1992, 89(7):3010-3014.

15. Benjamini Y, Hochberg Y: Controlling the false discovery rate: a practical and powerful approach to multiple testing. Journal of the Royal Statistical Society Series B 1995, 57:289-300.

16. Schoor O, Weinschehenk T, Hennenlotter J, Corvin S, Stenzl A, Rammensee HG, Stevenovic S: Moderate degradation does not preclude microarray analysis of small amounts of RNA. Biotechniques 2003, 35:1192-1201.

17. Schena M, Shalon D, Davis RW, Brown PO: Quantitative monitoring of gene expression patterns with a complementary DNA microarray. Science 1995, 270(5235):467-70.

18. Berger SL, Cooper LS: Very Short-Lived and Stable mRNAs from Resting Human Lymphocytes. PNAS 1975, 71(10):3873-3877.

\section{Submit your next manuscript to BioMed Central and take full advantage of:}

- Convenient online submission

- Thorough peer review

- No space constraints or color figure charges

- Immediate publication on acceptance

- Inclusion in PubMed, CAS, Scopus and Google Scholar

- Research which is freely available for redistribution

Submit your manuscript at www.biomedcentral.com/submit 